\title{
Óxidos de colesterol em alimentos
}

\section{Alfredo Tenuta-Filho*, Isabel Cristina Morales-Aizpurúa, Andréa Figueiredo Procópio de Moura, Sandra Emi Kitahara}

Departamento de Alimentos e Nutrição Experimental, Faculdade de Ciências Farmacêuticas, Universidade de São Paulo

*Correspondência:

A. Tenuta Filho

Departamento de Alimentos e

Nutrição Experimental

FCF-USP

Av. Prof. Lineu Prestes, 580. Bloco 14. 05508-900 - São Paulo - SP

E-mail: eetenuta@usp.br
A oxidação do colesterol em alimentos leva à formação de óxidos com características aterogênicas, citotóxicas, cancerígenas e mutagênicas, tornando obrigatório o exame criterioso da ingestão desses compostos. Leite integral em pó, manteiga, hambúrguer frito, bife grelhado e gema de ovo, fresca, frita e em pó foram quantificados em relação ao colesterol, $7 \alpha$-hidroxicolesterol ( $7 \alpha$ $\mathrm{OH}$ ), 7 $\beta$-hidroxicolesterol (7 $\beta$-OH), 7-cetocolesterol (7-ceto) e 25 hidroxicolesterol (25-OH), por HPLC. Os níveis de colesterol foram

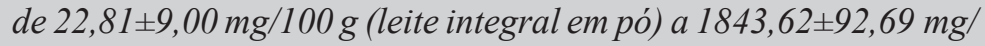
$100 \mathrm{~g}$ (gema em pó). O somatório dos óxidos derivados do carbono $7(7 \alpha-\mathrm{OH}, 7 \beta-\mathrm{OH}$ e 7 ceto) foi de $18,76 \pm 9,50 \mu \mathrm{g} / \mathrm{g}$ no leite integral

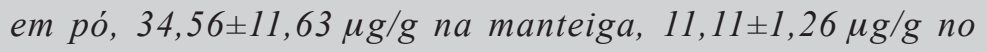
hambúrguer frito e $22,84 \pm 8,43 \mu \mathrm{g} / \mathrm{g}$ no bife grelhado. $O$ 7-ceto e 25-OH não foram detectados na gema fresca. $O$ 7-ceto ocorreu em

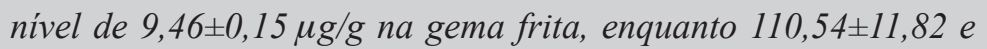
$112,67 \pm 13,52 \mu \mathrm{g} / \mathrm{g}$, respectivamente, de 7-ceto e 25-OH foram encontrados na gema em pó. Muitos dos resultados obtidos foram mais elevados que os relatados na literatura, sugerindo estudos mais detalhados visando indicar em que fase(s) (processamento el ou armazenamento) e em que grau a oxidação do colesterol ocorre nos alimentos.
Unitermos:

- Colesterol

- Oxidação de colesterol

- Óxidos de colesterol

- Alimentos

\section{INTRODUÇÃO}

A importância da oxidação do colesterol em alimentos se dá pelo fato de sua suscetibilidade química e, principalmente, em razão de os óxidos formados apresentarem propriedades biológicas deletérias, com características aterogênicas, citotóxicas, cancerígenas e mutagênicas, entre outras (Kendall et al., 1992; Peng et al., 1991; Petrakis et al., 1981; Sevanian, Peterson, 1986; Sporer et al., 1982).
Em razão disso, a formação e a ingestão desses compostos precisam ser profundamente examinadas. Não há, ainda, conhecimentos suficientes que permitam estabelecer limites seguros para a ocorrência desses óxidos em alimentos, o mesmo acontecendo em relação à ingestão desses compostos. Óxidos de colesterol não ocorrem em alimentos frescos, ou ocorrem em quantidade mínima, o que significa dizer que o processo oxidativo se dá durante o processamento e/ou armazenamento (Morales-Aizpurúa, Tenuta-Filho, 2002). 
Dezenas de óxidos de colesterol têm sido identificadas (Tai et al., 1999), sendo oito deles os mais freqüentemente encontrados em alimentos (mencionados por seus nomes comuns, sistemáticos ou triviais, respectivamente): 7-cetocolesterol, 3 $\beta$-hidroxicolest-5-en-7-ona ou 7-ceto; 20-hidroxicolesterol, colest-5-en-3 $\beta, 20$-diol ou 20-OH; 25-hidroxicolesterol, colest-5-en-3 $\beta, 25$-diol ou 25-OH; $7 \alpha$-hidroxicolesterol, colest-5-en-3 $\beta, 7 \alpha$-diol ou $7 \alpha-\mathrm{OH} ; 7 \beta$-hidroxicolesterol, colest-5-en-3 $\beta, 7 \beta$-diol ou $7 \beta-\mathrm{OH}$; colesterol-5,6 $\alpha$-epóxido, $5,6 \alpha$-epóxido- $5 \alpha-$ colestan-3 $\beta$-ol ou 5,6 $\alpha$-epóxido; colesterol-5,6 $6 \beta$-epóxido, $5,6 \beta$-epóxido- $5 \beta$-colestan- $3 \beta$-ol ou 5,6 6 -epóxido; e o colestanotriol, $5 \alpha$-colestan- $\beta, 5,6 \beta$-triol ou triol. O 7-ceto em geral tem sido encontrado em concentrações mais altas e, por isso, apontado como eventual indicador da oxidação do colesterol. Por outro lado, o 25-OH e o triol ocorrem em quantidades menores em relação aos demais óxidos mencionados (Addis, 1986; Gallina Toschi, Caboni, 1992; Guardiola et al., 1995; Lercker, Rodriguez-Estrada, 2000; Nielsen et al., 1996; Novelli et al., 1998; Peng et al., 1991; Pie et al., 1991; Zunin et al., 1995). Estes óxidos são todos citotóxicos e aterogênicos, em diferentes graus (Bösinger et al., 1993).

O objetivo deste artigo foi o de avaliar a oxidação do colesterol em alguns alimentos de expressivo consumo, através da ocorrência do 7 -ceto, $7 \alpha-\mathrm{OH}, 7 \beta-\mathrm{OH}$ e $25-\mathrm{OH}$.

\section{MATERIAL E MÉTODOS}

\section{Material}

O leite integral em pó, manteiga, hambúrguer frito, carne bovina (contra-filé), ovo e gema em pó foram adquiridos comercialmente em São Paulo - SP. O bife grelhado e o ovo frito foram preparados em laboratório.

O colesterol (5-cholesten-3 $\beta$-ol) (C8667), $7 \beta$ hidroxicolesterol (7 $\beta-\mathrm{OH})(\mathrm{H} 6891), 7$-cetocolesterol (7ceto) (C2394), 25-hidroxicolesterol (25-OH) (H1015) e o 6-cetocolestanol (3 $\beta$-hidroxy-5 $\alpha$-colestan-6-one) (K1250) foram adquiridos junto à Sigma Chemical Co, e o $7 \alpha-$ hidroxicolesterol $(7 \alpha-\mathrm{OH})(\mathrm{C} 6420)$ junto à Steraloids Inc. O hexano, da Merck (104391) ou Aldrich (27050-4), e o isopropanol, da Aldrich (27049-0), foram usados compondo a fase móvel, sendo ambos de grau HPLC. Os demais reagentes tinham grau analítico ACS compatível.

\section{Métodos}

O colesterol e óxidos de colesterol foram extraídos com clorofórmio/metanol (2:1), segundo a adaptação do método de Folch et al. (1957) feita por Csallany et al.
(1989). A fração de clorofórmio foi evaporada e o resíduo submetido à saponificação fria $\left(25-30^{\circ} \mathrm{C}\right)$, por 18-20 horas, com KOH $1 \mathrm{M}$, em metanol, conforme Chen, Chen (1994). A fração insaponificável foi extraída com $10 \mathrm{~mL}$ de hexano, por três vezes consecutivas.

Após a evaporação do hexano, ressuspensão do resíduo na fase móvel correspondente e a filtração em membrana $(0,45 \mu \mathrm{m})$ (Millex - Millipore), o extrato foi injetado no cromatógrafo. O equipamento usado foi um sistema de HPLC da marca Shimadzu, modelo SCL-10A $\mathrm{Ap}_{\mathrm{vp}}$, com detector de fotodiodos SPD-M10A $\mathrm{vp}_{\mathrm{vp}}$, bombas LC-10A $\mathrm{vp}$ injetor automático de amostras SIL-10AD $\mathrm{AD}_{\mathrm{v}} \mathrm{O}$ método utilizado foi o proposto por Csallany et al. (1989), através de coluna de sílica ( $\mu$ Porasil; 30 X 0,39 cm; Waters Associates), diâmetro do poro de $10 \mathrm{~mm}$, em fase normal. O monitoramento se deu entre 206 a $233 \mathrm{~mm}$ para detecção do colesterol e óxidos de colesterol. A fase móvel usada correspondeu à mistura de hexano e isopropanol $(\mathrm{v} / \mathrm{v})$ nas proporções de 93:7, para o 7-ceto, 6-cetocolestanol (padrão interno), $7 \alpha-\mathrm{OH}$ e $7 \beta-\mathrm{OH}$, e $97: 3$, para o $25-\mathrm{OH}$ e colesterol.

A identificação dos analitos se deu pelo tempo de retenção, em relação aos padrões correspondentes. A quantificação foi feita por padronização interna, pela área do pico. As curvas-padrão do colesterol e óxidos de colesterol foram estabelecidas entre 10 e $50 \mu \mathrm{g}$ e 0,1 a $0,5 \mu \mathrm{g}$, respectivamente, com coeficientes de correlação linear de $\mathrm{r}^{2}=0,999$. Os limites de quantificação foram de $3,62 \times 10^{-8} \mathrm{~g}$ para o colesterol, $2,22 \times 10^{-8} \mathrm{~g}$ para o $25-\mathrm{OH}$, $6,73 \times 10^{-9} \mathrm{~g}$ para o 7 -ceto, $1,03 \times 10^{-7} \mathrm{~g}$ para o $7 \alpha-\mathrm{OH}$ e $2,30 \times 10^{-7} \mathrm{~g}$ para o $7 \beta-\mathrm{OH}$, empregados segundo Long, Winefordner (1983). A recuperação dos analitos variou entre 94,0-99,9\%. Os dados foram submetidos à análise de variância (Anova) segundo o programa Instat versão 2.01 (GraphPad Software), com significância de 5\%.

\section{RESULTADOS E DISCUSSÃO}

\section{Colesterol}

O colesterol foi quantificado em valores médios entre 23 e $96 \mathrm{mg} / 100 \mathrm{~g}$, no leite integral em pó, manteiga, hambúrguer frito e bife grelhado (Tabelas I a IV). Na gema de ovo, fresca, frita ou em pó, o colesterol foi encontrado entre $1033-1844 \mathrm{mg} / 100 \mathrm{~g}$. Significativa variabilidade foi observada entre as amostras correspondentes a cada um dos alimentos das Tabelas I a IV, a considerar pelo coeficiente de variação, notadamente no leite integral em pó (Tabela I) e no bife grelhado (Tabela IV).

A oxidação do colesterol foi evidenciada nesses alimentos, com exceção da gema fresca, traduzida pela pre- 
sença do $7 \alpha-\mathrm{OH}, 7 \beta-\mathrm{OH}$ e 7 -Ceto, derivados do carbono 7 , e do $25-\mathrm{OH}$, oriundo da cadeia lateral. A variabilidade dos óxidos observada em relação às amostras de cada alimento foi maior que a verificada para o colesterol (Tabelas I a IV).

\section{Óxidos de Colesterol}

\section{Leite Integral em Pó}

A ocorrência de $7 \alpha-\mathrm{OH}, 7 \beta-\mathrm{OH}$ e 7 -ceto deu-se totalizando $18,76 \pm 9,50 \mu \mathrm{g} / \mathrm{g}$ nas amostras de leite integral em pó, ou 0,11 $\pm 0,09 \%$ em relação ao colesterol. Eventual predominância do 7-ceto sobre os demais óxidos não foi confirmada (Tabela I).

A concentração média total dos três óxidos examinados foi cerca de 9,4-94,0 vezes maior que a concentração total dos óxidos de colesterol observada em leites em pó recentemente fabricados $(0,2-2,0 \mu \mathrm{g} / \mathrm{g})$ (Appelqvist, 1996). A suspeição de que as amostras estudadas poderiam estar em franca oxidação relativa ao colesterol é reforçada também com base em Angulo et al. (1997), Lercker, Rodriguez-Estrada (2000) e Nourooz-Zadeh, Appelqvst (1988), que chegaram a quantidades significativamente menores que as mostradas na Tabela I, relatando-as em até $2,5 \mu \mathrm{g} / \mathrm{g}$ para cada um dos referidos óxidos, tanto em leite integral em pó como em leite desnatado em pó.

Segundo Appelqvist (1996), amostras comerciais de leite em pó variam quanto ao teor de óxidos de colesterol como reflexo do processo de desidratação e/ou armazenamento. Angulo et al. (1997) comprovaram a ocorrência dessa oxidação pela relação direta com condições de armazenagem estudadas - de até 12 meses, na ausência de luz, sob nitrogênio ou ar atmosférico, a $32{ }^{\circ} \mathrm{C}$ ou $55^{\circ} \mathrm{C}$ em leite desnatado em pó e em leite integral em pó, estudo no qual o 7-ceto foi o óxido de maior formação entre os analisados.

Valores maiores, no entanto, mas ainda menores que os encontrados no presente trabalho, foram relatados por Sarantinos et al. (1993) em amostras comerciais de leite integral em pó $(2-14 \mu \mathrm{g} / \mathrm{g}$ de $7 \alpha-\mathrm{OH}, 2-3 \mu \mathrm{g} / \mathrm{g}$ de $7 ß-\mathrm{OH}$ e $5 \mu \mathrm{g} / \mathrm{g}$ de 7 -ceto). Foram detectados, também, o 22-cetocolesterol (3-10 $\mu \mathrm{g} / \mathrm{g}), 20-\mathrm{OH}(6-11 \mu \mathrm{g} / \mathrm{g})$ e triol $(1 \mu \mathrm{g} / \mathrm{g})$.

A ocorrência da oxidação do colesterol durante a armazenagem do leite em pó é um aspecto de extrema importância, pelo fato de o produto ter prazo de validade comercial bastante longo, de 18 meses em alguns casos. Ao mesmo tempo, o seu uso integrando formulações alimentares que sofrerão aquecimento posterior, como é comum ocorrer, preocupa também pela oxidação adicional do colesterol.

\section{Manteiga}

Foi constatado na manteiga examinada um somatório de óxidos de 34,56 $\pm 11,63 \mu \mathrm{g} / \mathrm{g}, 0,04 \pm 0,02 \% \mathrm{em}$ relação ao colesterol, e verificado um valor significativamente menor para o $7 \alpha-\mathrm{OH}$ em relação aos dois outros óxidos (Tabela II).

Pie et al. (1990) analisaram amostras de manteiga, frescas ou não. No produto fresco detectaram apenas traços de 7-ceto; no aquecido a $180{ }^{\circ} \mathrm{C} / 20$ minutos: $3,93 \pm$ $0,04 \mu \mathrm{g} / \mathrm{g}$ de $7 \alpha-\mathrm{OH}, 4,58 \pm 0,32 \mu \mathrm{g} / \mathrm{g}$ de $7 \beta-\mathrm{OH}$ e $8,63 \pm$ $0,01 \mu \mathrm{g} / \mathrm{g}$ de 7 -ceto (total de $17,14 \mu \mathrm{g} / \mathrm{g}$ ), e ainda $2,90 \pm$ 0,31 e 7,26 $\pm 0,25 \mu \mathrm{g} / \mathrm{g}$, respectivamente de 5,6 $\alpha$-epóxido e 5,6 $\beta$-epóxido; e, nos mantidos congelados $\left(-20^{\circ} \mathrm{C}\right)$, por 3 ou 6 meses, e depois aquecidos a $180{ }^{\circ} \mathrm{C} / 20$ minutos: $4,13 \pm 0,07$ e $8,88 \pm 0,34 \mu \mathrm{g} / \mathrm{g}$ de $7 \alpha-\mathrm{OH}, 5,73 \pm 0,15 \mathrm{e}$ $14,88 \pm 0,40 \mu \mathrm{g} / \mathrm{g}$ de $7 \beta-\mathrm{OH}$ e $10,78 \pm 0,23$ e $14,35 \pm$ $0,42 \mu \mathrm{g} / \mathrm{g}$ de 7 -ceto (totais de 20,64 e 38,11 $\mu \mathrm{g} / \mathrm{g}$ ), além de $2,83 \pm 0,04$ e 7,40 $\pm 0,65 \mu \mathrm{g} / \mathrm{g}$ de 5,6 $\alpha$-epóxido e 9,21 \pm 0,03 e 18,45 $\pm 2,07 \mu \mathrm{g} / \mathrm{g}$ de 5,6 6 -epóxido. Com isso o nível de oxidação do colesterol na manteiga analisada no presente trabalho foi igual ou superior à submetida a congelamento e a forte aquecimento prévios, estudada por Pie et al. (1990).

TABELA I - Colesterol (mg/100 g) e óxidos de colesterol $(\mu \mathrm{g} / \mathrm{g})$ em leite integral em pó $(\mathrm{n}=5)$

\begin{tabular}{lcccccc}
\hline & Colesterol & $7 \alpha-\mathrm{OH}$ & $7 \beta-\mathrm{OH}$ & 7 -ceto & $\sum$ óxidos & $\%^{*}$ \\
\hline $\mathrm{Mi}$ & 9,27 & 0,65 & 5,42 & 3,68 & 10,92 & 0,03 \\
$\mathrm{Mx}$ & 34,16 & 15,51 & 14,49 & 17,7 & 30,92 & 0,23 \\
$\mathrm{M}$ & 22,81 & $5,03^{\mathrm{a}}$ & $9,78^{\mathrm{a}}$ & $7,74^{\mathrm{a}}$ & 18,76 & 0,11 \\
$\mathrm{DP}$ & 9,00 & 6,33 & 3,93 & 6,68 & 9,50 & 0,09 \\
$\mathrm{CV}$ & 39,46 & 125,70 & 40,20 & 86,41 & 50,65 & 84,46 \\
\hline
\end{tabular}

Mi = Mínimo; $\mathrm{Mx}=$ Máximo; $\mathrm{M}=$ Médio; $\mathrm{DP}=$ Desvio-Padrão; $\mathrm{CV}=$ Coeficiente de Variação (\%); $\Sigma$ óxidos $=(7 \alpha-$ $\mathrm{OH})+(7 \beta-\mathrm{OH})+(7$-ceto $) ; \% *=\sum$ óxidos em relação ao colesterol. Letras superescritas iguais indicam diferenças estatísticas não-significativas. 
TABELA II - Colesterol (mg/100 g) e óxidos de colesterol $(\mu \mathrm{g} / \mathrm{g})$ em manteiga $(\mathrm{n}=5)$.

\begin{tabular}{lcccccc}
\hline & Colesterol & $7 \alpha-\mathrm{OH}$ & $7 \beta-\mathrm{OH}$ & 7 -ceto & $\sum$ óxidos & $\%^{*}$ \\
\hline $\mathrm{Mi}$ & 74,62 & 1,85 & 10,43 & 6,76 & 20,19 & 0,02 \\
$\mathrm{Mx}$ & 106,69 & 6,95 & 12,61 & 39,31 & 51,73 & 0,07 \\
$\mathrm{M}$ & 95,66 & $3,97^{\mathrm{a}}$ & $11,60^{\mathrm{b}}$ & $18,98^{\mathrm{b}}$ & 34,56 & 0,04 \\
$\mathrm{DP}$ & 12,91 & 2,12 & 0,85 & 12,43 & 11,63 & 0,02 \\
$\mathrm{CV}$ & 13,50 & 53,52 & 7,32 & 65,50 & 33,66 & 48,99 \\
\hline
\end{tabular}

Mi = Mínimo; $\mathrm{Mx}=$ Máximo; $\mathrm{M}=$ Médio; $\mathrm{DP}=$ Desvio-Padrão; $\mathrm{CV}=$ Coeficiente de Variação (\%); $\Sigma$ óxidos $=(7 \alpha-$ $\mathrm{OH})+(7 \beta-\mathrm{OH})+(7-$ ceto $) ; \% *=\sum$ óxidos em relação ao colesterol. Letras superescritas diferentes indicam diferenças estatísticas significativas.

Resultados originalmente expressos em $\mu \mathrm{g} / \mathrm{g}$ de lípides foram também relatados na literatura, em relação aos quais (recalculados em $\mu \mathrm{g} / \mathrm{g}$ de manteiga, com base num teor lipídico de $80 \%$ ) os da Tabela II são igualmente superiores. Nielsen et al. (1996) encontraram 0,24-0,96 $\mu \mathrm{g} / \mathrm{g}$ de $7 \alpha-\mathrm{OH}, 0,21-0,60 \mu \mathrm{g} / \mathrm{g}$ de $7 \beta-\mathrm{OH}, 1,04-2,32 \mu \mathrm{g} / \mathrm{g}$ de $7-$ ceto, $0,08-0,30 \mu \mathrm{g} / \mathrm{g}$ de 5,6 $\alpha$-epóxido, $0,15-0,23 \mu \mathrm{g} / \mathrm{g}$ de 5,63-epóxido e $0,00-0,19 \mu \mathrm{g} / \mathrm{g}$ de triol, em manteiga armazenada por 32 semanas $/-18^{\circ} \mathrm{C}, 11$ semanas $/ 4^{\circ} \mathrm{C}$ e 13 semanas a $20^{\circ} \mathrm{C}$, respectivamente, enquanto Lee et al. (1998) detectaram $2,88 \pm 0,42 \mu \mathrm{g} / \mathrm{g}$ de 7 -ceto e $1,36 \pm 0,03 \mu \mathrm{g} / \mathrm{g}$ de $5,6 \beta$-epóxido, e um teor de $7 \beta-\mathrm{OH}$ de $16,59 \pm 2,06 \mu \mathrm{g} / \mathrm{g}$, que excepcionalmente superou os da Tabela II.

A Tabela II caracteriza a oxidação do colesterol nas amostras estudadas. Isso inclui levar em conta o potencial de oxidação adicional da manteiga, quando esta é usada em processos de fritura, em substituição ao óleo, ou fazendo parte de formulações alimentares que venham a ser aquecidas posteriormente, como é comum ocorrer, à semelhança do leite em pó.

\section{Hambúrguer Frito}

No hambúrguer frito foi encontrado total de óxidos de $11,11 \mu \mathrm{g} / \mathrm{g}$, correspondente a $0,3 \pm 0,01 \%$ do colesterol presente (Tabela III).

Os teores de 7-ceto indicados para o hambúrguer frito, de 3,45-5,33 $\mu \mathrm{g} / \mathrm{g}$, superaram os mencionados por De Vore (1988) e Rodriguez-Estrada et al. (1997) (0,06$3,48 \mu \mathrm{g} / \mathrm{g})$, tanto para hambúrguer cru como para processados por diferentes maneiras, inclusive por fritura. As quantidades da Tabela III referentes ao $7 \alpha-\mathrm{OH}(0,79$ $2,79 \mu \mathrm{g} / \mathrm{g}$ ) foram menores que a apontada para hambúrguer cozido $(72 \mu \mathrm{g} / \mathrm{g})$, por Higley, Taylor (1986), ao passo que em relação aos valores de $7 ß-\mathrm{OH}(5,09-5,61 \mu \mathrm{g} / \mathrm{g})$ foram maiores que o relatado pelos mesmos autores para o hambúrguer cru $(3,6 \mu \mathrm{g} / \mathrm{g})$.

\section{Bife Grelhado}

O valor médio total dos três óxidos encontrados no bife grelhado atingiu $22,84 \pm 8,43 \mu \mathrm{g} / \mathrm{g}$, ou $0,07 \pm 0,04 \%$ do colesterol, com predominância do 7-ceto sobre os demais (Tabela IV).

O conteúdo de 7-ceto verificado, de 16,92 $\pm 6,82 \mu \mathrm{g} / \mathrm{g}$, foi bem maior que os de Zubillaga, Maerker (1991), relativos à carne bovina estocada a $0-4{ }^{\circ} \mathrm{C} / 7-21$ dias, 1,02 $2,55 \mu \mathrm{g} / \mathrm{g}$, produto que também continha $0,13-0,37 \mu \mathrm{g} / \mathrm{g}$ de

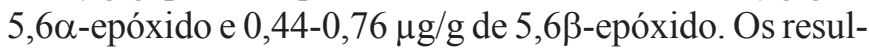
tados da Tabela IV também superaram os de Pie et al.

TABELA III - Colesterol (mg/100 g) e óxidos de colesterol $(\mu \mathrm{g} / \mathrm{g})$ em hambúrguer frito $(\mathrm{n}=5)$.

\begin{tabular}{lcccccc}
\hline & Colesterol & $7 \alpha-\mathrm{OH}$ & $7 \beta-\mathrm{OH}$ & 7 -ceto & $\sum$ óxidos & $\%^{*}$ \\
\hline $\mathrm{Mi}$ & 21,47 & 0,79 & 5,09 & 3,45 & 9,67 & 0,03 \\
$\mathrm{Mx}$ & 45,89 & 2,79 & 5,61 & 5,33 & 12,89 & 0,05 \\
$\mathrm{M}$ & 36,23 & $1,46^{\mathrm{a}}$ & $5,43^{\mathrm{b}}$ & $4,23^{\mathrm{c}}$ & 11,11 & 0,03 \\
$\mathrm{DP}$ & 9,60 & 0,79 & 0,21 & 0,77 & 1,26 & 0,01 \\
$\mathrm{CV}$ & 26,49 & 54,52 & 3,84 & 18,29 & 11,38 & 26,65 \\
\hline
\end{tabular}

Mi = Mínimo; $\mathrm{Mx}=$ Máximo; $\mathrm{M}=$ Médio; $\mathrm{DP}=$ Desvio-Padrão; $\mathrm{CV}=$ Coeficiente de Variação $(\%) ; \Sigma$ óxidos $=(7 \alpha-$ $\mathrm{OH})+(7 \beta-\mathrm{OH})+(7-$ ceto $) ; \% *=\sum$ óxidos em relação ao colesterol. Letras superescritas diferentes indicam diferenças estatísticas significativas. 
TABELA IV - Colesterol (mg/100g) e óxidos de colesterol $(\mu \mathrm{g} / \mathrm{g})$ em bife grelhado $(\mathrm{n}=5)$.

\begin{tabular}{lcccccc}
\hline & Colesterol & $7 \alpha-\mathrm{OH}$ & $7 \beta-\mathrm{OH}$ & 7 -ceto & $\sum$ óxidos & $\%^{*}$ \\
\hline $\mathrm{Mi}$ & 24,86 & 0,40 & 2,55 & 9,01 & 14,97 & 0,03 \\
$\mathrm{Mx}$ & 66,85 & 3,00 & 5,67 & 26,64 & 35,31 & 0,12 \\
$\mathrm{M}$ & 41,95 & $1,25^{\mathrm{a}}$ & $4,67^{\mathrm{b}}$ & $16,92^{\mathrm{c}}$ & 22,84 & 0,07 \\
$\mathrm{DP}$ & 16,56 & 1,01 & 1,22 & 6,82 & 8,43 & 0,04 \\
$\mathrm{CV}$ & 39,49 & 80,61 & 26,04 & 40,29 & 36,90 & 66,32 \\
\hline
\end{tabular}

Mi = Mínimo; $\mathrm{Mx}=$ Máximo; $\mathrm{M}=$ Médio; $\mathrm{DP}=$ Desvio-Padrão; $\mathrm{CV}=$ Coeficiente de Variação (\%); $\Sigma$ óxidos $=7(\alpha-$ $\mathrm{OH})+(7 \beta-\mathrm{OH})+(7-$ ceto $) ; \% *=\sum$ óxidos em relação ao colesterol. Letras superescritas diferentes indicam diferenças estatísticas significativas.

(1991), principalmente os de 7-ceto, em relação ao bife cru e frito durante 3 e 10 minutos. Níveis de $0,33,0,51$ e $0,58 \mu \mathrm{g} / \mathrm{g}$ de $7 \alpha-\mathrm{OH}, 0,34,0,58$ e $0,67 \mu \mathrm{g} / \mathrm{g}$ de $7 \beta-\mathrm{OH}$ e $1,12,1,66$ e $2,11 \mu \mathrm{g} / \mathrm{g}$ de 7 -ceto, além de $0,14,0,23$ e $0,34 \mu \mathrm{g} / \mathrm{g}$ de $25-\mathrm{OH}$, foram detectados nesses produtos, respectivamente.

Os resultados da Tabela IV são, ainda, muito maiores que os de outros autores, como os de Hwang, Maerker (1993), que observaram $0,21 \mu \mathrm{g} / \mathrm{g}$ de 7 -ceto em bife cru. No bife cru armazenado a 0 e 4 dias $/ 4^{\circ} \mathrm{C}$ Engeseth, Gray (1994) encontraram 1,4 e $0,7 \mu \mathrm{g} / \mathrm{g}$ de $7 \beta-\mathrm{OH}$, e ainda 0 $5,2 \mu \mathrm{g} / \mathrm{g}$ de 5,6 6 -epóxido, respectivamente. Depois de assado em forno convencional, os autores detectaram 3,1 e $4,8 \mu \mathrm{g} / \mathrm{g}$ de $7 \beta-\mathrm{OH}$ e traços- $4,8 \mu \mathrm{g} / \mathrm{g}$ de 7 -ceto, e ainda traços-7,7 $\mu \mathrm{g} / \mathrm{g}$ de 5,6ß-epóxido, respectivamente.

\section{Gema de Ovo}

Amostras ( $n=3)$ de gema de ovo, fresca, frita ou em pó, foram analisadas em relação ao 7-ceto e 25-OH. Como era previsível ocorrer, e concordando com Sarantinos et al. (1993), não foi observada a presença desses óxidos no produto fresco. A gema frita continha 9,46 $\pm 0,15 \mu \mathrm{g} / \mathrm{g}$ de 7-ceto, 0,09 \% em relação ao colesterol $(1033,38 \pm$ $14,81 \mathrm{mg} / 100 \mathrm{~g}$ ), não sendo confirmada a presença de 25$\mathrm{OH}$. Os autores mencionados não detectaram 7-ceto e 25$\mathrm{OH}$ em ovo frito. Os níveis de 7-ceto e $25-\mathrm{OH}$ encontrados na gema em pó foram, respectivamente, de 110,54 \pm 11,82 e $112,67 \pm 13,52 \mu \mathrm{g} / \mathrm{g}$, que somados corresponderam a $1,2 \%$ do colesterol $(1843,62 \pm 92,69 \mathrm{mg} / 100 \mathrm{~g})$. O total de óxidos encontrados em diferentes alimentos tem sido na ordem de 1-2 a 10\% relativo ao colesterol presente (Paningvait et al.,1995). Para gema em pó Sarantinos et al. (1993) relataram $35 \pm 35 \mu \mathrm{g} / \mathrm{g}$ de 7-ceto, valor mais baixo do observado no presente trabalho, mas não detectaram 25-OH.

Sarantinos et al. (1993) analisaram o ovo fresco, refrigerado $\left(4^{\circ} \mathrm{C} / 6\right.$ semanas $)$, frito, cozido e em pó, além da gema em pó, em relação ao $7 \alpha-\mathrm{OH}, 7 \beta-\mathrm{OH}, 7$-ceto, 20 -

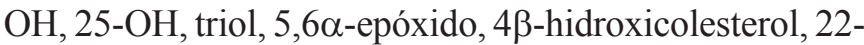
cetocolesterol $(n=3)$. Com exceção do ovo fresco ou refrigerado, que não continham os citados óxidos, nos demais produtos o total dos óxidos de colesterol ( $84 \pm 20$ a 1132 $\pm 91 \mu \mathrm{g} / \mathrm{g}$ ) encontrados relativo ao nível do colesterol correspondente foi de $2,1 \pm 0,5$ a $4,9 \pm 0,4 \%$. O $25-\mathrm{OH}$ não foi detectado nos produtos analisados.

Como no caso do leite em pó, a produção e o armazenamento longo da gema em pó (seis meses) podem propiciar a oxidação do colesterol. Esse aspecto tem a importância ainda aumentada, quando se considera que a gema em pó compõe como matéria-prima a formulação de outros alimentos, estando sujeita a processamento e armazenamento posteriores.

\section{CONCLUSÕES}

Os resultados observados de um modo geral foram mais elevados que os previamente relatados, ao mesmo tempo em que provavelmente subestimam a oxidação do colesterol, na medida em que somente parte dos óxidos de maior ocorrência foi quantificada. Esses resultados sugerem que os alimentos aqui analisados, e também outros, sejam investigados mais detalhadamente. É importante definir em que fase(s) do processamento e/ou armazenamento ocorre a oxidação do colesterol e as possibilidades de sua inibição, exercendo, ainda, o controle do alimento até o momento exato do consumo.

\section{ABSTRACT}

\section{Cholesterol oxides in foods}

Cholesterol oxidation in foods yields oxides that show cytotoxic, atherogenic, mutagenic and carcinogenic properties suggesting that the intake of these compounds 
should then be examined criteriously. Whole milk powder, butter, fried hamburger, grilled beef and egg yolk, fresh, fried and powder, were analyzed in relation to cholesterol and their oxides $7 \alpha$-hydroxycholesterol $(7 \alpha-O H), 7 \beta$ hydroxycholesterol (7 $\beta$-OH), 7-ketocholesterol (7-keto) and 25-hydroxycholesterol (25-OH), by HPLC. Cholesterol values varied from $22.81 \pm 9.00 \mathrm{mg} / 100 \mathrm{~g}$ (whole milk powder) to $1843.62 \pm 92.69 \mathrm{mg} / 100 \mathrm{~g}$ (egg yolk powder). The total values of the oxides derived from carbon-7 ( $7 \alpha$ $\mathrm{OH}, 7 \beta-\mathrm{OH}$ and 7 -keto) amounted $18.76 \pm 9.50 \mu \mathrm{g} / \mathrm{g}$ for whole milk powder, $34.56 \pm 11.63 \mu \mathrm{g} / \mathrm{g}$ for butter, 11.11 $\pm 1.26 \mu \mathrm{g} / \mathrm{g}$ for fried hamburger and $22.84 \pm 8.43 \mu \mathrm{g} / \mathrm{g}$ for grilled beef. In fresh egg yolk neither 7-keto nor 25-OH were found. Levels of $9.46 \pm 0.15 \mu \mathrm{g} / \mathrm{g}$ for 7-keto were found in fried egg yolk and $110.54 \pm 11.82 \mu \mathrm{g} / \mathrm{g}$ for 7-keto and $112.67 \pm 13.52 \mu \mathrm{g} / \mathrm{g}$ for $25-\mathrm{OH}$ in egg yolk powder. Many results here obtained were greater than those previously reported suggesting more detailled studies should be performed mainly in relation to food processing and storage.

UNITERMS: Cholesterol. Cholesterol oxidation. Cholesterol oxides. Foods.

\section{AGRADECIMENTOS}

Ao CNPq e à FAPESP, pelo apoio ao projeto de pesquisa, e à universitária Luciene Fagundes Lauer Macedo, pelo auxílio técnico prestado.

\section{REFERÊNCIAS BIBLIOGRÁFICAS}

ADDIS, P. B. Occurrence of lipid oxidation products in foods. Food Chem. Toxic., Oxford, v.24, n.10/11, p.1021-1030, 1986.

ANGULO, A. J.; ROMERA, J. M.; RAMIREZ, M.; GIL, A. Determination of cholesterol oxides in dairy products: effect of storage conditions. J. Agric. Food Chem., Columbus, v.45, p.4318-4323, 1997.

APPELQVIST, L. A. Oxidized sterols. Bull. Int. Dairy Fed., Brussels, v.315, p.52-58, 1996.

BÖSINGER, S.; LUF, W.; BRANDL, E. Oxysterols: their occurrence and biological effects. Int. Dairy J., Oxford, v.3, p.1-33, 1993.

CHEN, B. H.; CHEN, Y. C. Evaluation of the analysis of cholesterol oxides by liquid chromatography. $J$. Chromatogr., A, Amsterdam, v.661, p.127-136, 1994.
CSALLANY, A. S.; KINDOM, S. E.; ADDIS, P. B.; LEE, J.H. HPLC method for quantitation of cholesterol and four of its major oxidation products in muscle and liver tissues. Lipids, Champaign, v.24, n.7, p.645-651, 1989.

DE VORE, V. R. TBA values and 7-ketocholesterol in refrigerated raw and cooked ground beef. J. Food Sci., Chicago, v.53, n.4, p.1058-1061, 1988.

ENGESETH, N. J.; GRAY, J. I. Cholesterol oxidation in muscle tissue. Meat Sci., Oxford, v.36, p.309-320, 1994.

FOLCH, J.; LEES, M.; STANLEY, S. G. H. A simple method for the isolation and purification of total lipides from animal tissues. J. Biol. Chem., Bethesda, v.226, p.497509, 1957.

GALLINA TOSCHI, T.; CABONI, M. F. Cholesterol oxides: biological behaviour and analytical determination. Ital. J. Food Sci., Pinerolo, v.4, p.223-228, 1992.

GUARDIOLA, F.; CODONY, R.; RAFECAS, M.; BOATELLA, J. Formación de derivados oxidados del colesterol en alimentos. Grasas Aceites, Sevilla, v.46, n.3, p.202-212, 1995 .

HIGLEY, N. A.; TAYLOR, S. L. Cholesterol oxides in processed meats. MeatSci., Oxford, v.16,p.175-188, 1986.

HWANG, K. T.; MAERKER, G. Quantitation of cholesterol oxidation products in unirradiated and irradiated meats. J. Am. Oil Chem. Soc., Champaign, v.70, n.4, p.371-375, 1993.

KENDALL, C. W.; KOO, M.; SOKOLOFF, E.; KESAVARAO, G. Effects of dietary oxidized cholesterol on azoxymethane induced colonic preneoplasia in mice. Cancer Lett., Shannon, v.66, p.241-248, 1992.

LEE, J. I.; KANG, S. N.; BYUN, J. S.; LEE, M. Identification and quantification of cholesterol oxides in foods. Korean J. Anim. Sci., Seoul, v.40, n.5, p.529-538, 1998.

LERCKER, G.; RODRIGUEZ-ESTRADA, M. T. Cholesterol oxidation: presence of 7-ketocholesterol in different food products. J. Food Compos. Anal., Orlando, v.13, p.625-631, 2000.

LONG, G. L.; WINEFORDNER, J. D. Limit of detection: a closer look at the IUPAC definition. Anal. Chem., Columbus, v.55, n.7, p.712A -724A, 1983. 
MORALES-AIZPURÚA, I. C.; TENUTA-FILHO, A. Óxidos de colesterol: ocorrência em alimentos, formação e efeitos biológicos. Rev. Bras. Cienc. Farm., São Paulo, v.38, n.4, p.431-442, 2002.

NIELSEN, J. H.; OLSEN, C. E.; JENSEN, C.; SKIBSTED, L. H. Cholesterol oxidation in butter and dairy spread during storage. J. Dairy Res., Cambridge, v.63, p.159167, 1996.

NOUROOZ-ZADEH, J.; APPELQVIST, L. A. Cholesterol oxides in Swedish foods and foods ingredients: milk powder products. J. Food Sci., Chicago, v.53, n.1, p.7479, 1988.

NOVELI, E.; ZANARDI, E.; GHIRETTI, G. P.; COMPANINI, G.; DAZZI, G.; MADARENA, G.; CHIZZOLINI, R. Lipid and cholesterol oxidation in frozen stored pork, salame Milano and mortadella. Meat Sci., Oxford, v.48, n.1/2, p.29-40, 1998.

PANIANGVAIT, P.; KING, A. J.; JONES, A. D.; GERMAN, B. G. Cholesterol oxides in foods of animal origin. $J$. Food Sci., Chicago, v.60, n.6, p.1159-1174, 1995.

PENG, S.-K.; HU, B.; MORIN, R. J. Angiotoxicity and atherogenicity of cholesterol oxides. J. Clin. Lab. Anal., New York, v.5, p.144-152, 1991.

PETRAKIS, N. L.; GRUENKE, L. D.; CRAIG, J. L. Cholesterol and cholesterol epoxides in nipple aspirates of human breast fluid. Cancer Res., Philadelphia, v.41, p.2563-2565, 1981.

PIE, J. E.; SPAHIS, K.; SEILLAN, C. Cholesterol oxidation in meat products during cooking and frozen storage. $J$. Agric. Food Chem., Columbus, v.39, n.2, p.250-254, 1991.

PIE, J.E.; SPAHIS, K.; SEILLAN, C. Evaluation of oxidative degradation of cholesterol in food and food ingrediens: Identification and quantification of cholesterol oxides. $J$. Agric. Food Chem., Columbus, v.38, n.4, p.973-979, 1990.
RODRIGUEZ-ESTRADA, M. T.; PENAZZI, G.; CABONI, M. F.; BERTACCO, G.; LERCKER, G. Effect of different cooking methods on some lipid and protein components of hamburgers. Meat Sci., Oxford, v.45, n.3, p.365-375, 1997.

SARANTINOS, J.; O’DEA, K.; SINCLAIR, A. J. Cholesterol oxides in Australian foods: identification and quantification. Food Aust., North Sydney, v.45, n.10, p.485-490, 1993.

SEVANIAN, A.; PETERSON, A. R. The cytotoxic and mutagenic propertiers of cholesterol oxidation products. Food Chem. Toxicol., Oxford, v.24, n.10/11, p.11031110, 1986.

SPORER, A.; BRILL, D. R.; SCHAFFNER, C. P. Epoxycholesterols in secretions and tissue of normal, benign, and cancerous human prostate glands. Urology, New York, v.20, p.244-250, 1982.

TAI, C.-Y.; CHEN, Y. C.; CHEN, B. H. Analysis, formation and inhibition of cholesterol oxidation products in foods: an overview (Part 1). J. Food Drug Anal., Nankang, v.7, n. 4, p. $243-257,1999$.

ZUBILLAGA, M. P.; MAERKER, G. Quantification of three cholesterol oxidation products in raw meat and chicken. J. Food Sci., Chicago, v.56, n.5, p.1194-1196 e 1202, 1991.

ZUNIN, P.; EVANGELISTI, F.; CABONI, M. F.; PENAZZI, G.; LERCKER, G.; TISCORNIA, E. Cholesterol oxidation in baked foods containing fresh and powdered eggs. J. Food Sci., Chicago, v.60, n.5, p.913-916, 1995.

Recebido para publicação em 18 de março de 2003. 